\title{
The shrinking chip
}

\section{The delivery of a 13.5-nm light source to one of the world's leading producers of lithographic equipment suggests that a new era of silicon chip manufacturing may be in sight.}

Silicon chip manufacturers trying to keep up with Moore's law are most likely breathing a sigh of relief that the US firm Cymer has recently delivered an extreme ultraviolet (EUV) light source to ASML (the Dutch provider of lithography systems for semiconductor manufacturing) for testing ${ }^{1}$.

According to ASML, it is now installing the 13.5-nm-wavelength source at its headquarters in Veldhoven, after which it will test a next-generation EUV lithography scanner that should allow chip manufacturers to define features smaller than $22 \mathrm{~nm}$. The Dutch firm says that it has received orders for five EUV lithographic systems from manufacturers of memory and logic chips, and plans to start delivery in 2010.

Although Moore's law ${ }^{2}$ - the empirical prediction made by Intel co-founder Gordon Moore in 1965 that says the number of transistors within an integrated circuit grows exponentially with time, doubling approximately every two years was undoubtedly a driving force behind the computer revolution, it is a constant source of stress for chip manufacturers. Indeed, in recent years there have been fears that fabrication technology would not be able to keep up - failure to meet Moore's law would highlight an undesirable slowing of progress in chip technology.

In common with the optical data storage industry, one of the key ways of achieving Moore's law over recent years has been to shorten the wavelength of light used, allowing the projection of smaller feature sizes onto the silicon wafer surface. In the data disk industry, the challenge is reading and writing ever-smaller 'pits' of digital data on disk surfaces - the required wavelength has almost halved from the near-infrared (CD, $780 \mathrm{~nm})$ to the blue ('Blu-Ray', $405 \mathrm{~nm}$ ). In chip lithography the source wavelength has always been in the ultraviolet, but over the past 20 years this has also halved from $365 \mathrm{~nm}$ to the current value of $193 \mathrm{~nm}$, resulting in socalled deep ultraviolet (DUV) lithography. Furthermore, the design rule (which defines the minimum achievable feature size) of chip lithography has reduced from around $0.5 \mu \mathrm{m}$ to just $22-35 \mathrm{~nm}$. The hope is that EUV lithography will

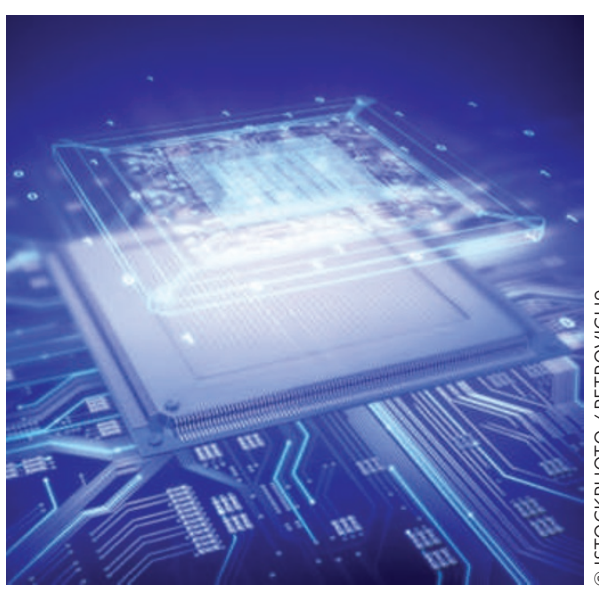

reported reaching a resolution of $15 \mathrm{~nm}$ using quadruple patterning at $193 \mathrm{~nm}$ (ref. 4). However, the costs associated with the extra processing steps are considerable, casting doubt over how cost-effective massproduction will be.

In this context, news that the source developer Cymer has shipped a fully integrated laser-produced plasma EUV light source to ASML gives cause for cautious optimism that a commercial EUV lithography system (and in principle another ten years of Moore's law) is in sight. Of course, developing the source is only part of the challenge, but it is still a significant achievement. In the source developed by Cymer, microscopic droplets of molten tin are fired through a vacuum chamber, tracked individually and then vapourized by a high-power pulsed infrared laser at a repetition rate of $50 \mathrm{kHz}$. The result is a high-temperature tin plasma that radiates light at $13.5 \mathrm{~nm}$. A large EUV mirror collects the emission and directs it into a scanner where it passes through a photomask, before being projected onto the surface of a silicon wafer through a complex set of image-reducing mirrors.

It will be interesting to see if the EUV lithography systems are delivered next year as originally planned. ASML certainly appears to be confident. "Cymer and ASML are committed to bringing EUV lithography to production reality for chip makers worldwide," said Martin van den Brink, Executive Vice President of Products and Technology at ASML. "EUV is by far the most cost-effective volume lithography technology for producing powerful next-generation semiconductors, and our achievements show how the equipment industry is making huge strides to introduce the first production-ready EUV lithography tools in 2010. This revolutionary new architecture provides chip manufacturers with the technology to extend the semiconductor shrinking process for another decade at least."

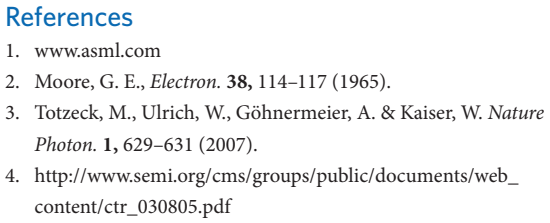

